\title{
Prevalence of Intestinal Parasites and Associated Risk Factors Among Schoolchildren of Homesha District (Woreda) in Benishangul-Gumuz Regional State, Western Ethiopia
}

\author{
Gebremichael Gebretsadik \\ Department of Biology, Assosa University, Assosa, Ethiopia
}

Email address:

mikigebre73@gmail.com

To cite this article:

Gebremichael Gebretsadik. Prevalence of Intestinal Parasites and Associated Risk Factors Among Schoolchildren of Homesha District (Woreda) in Benishangul-Gumuz Regional State, Western Ethiopia. Journal of Family Medicine and Health Care.

Vol. 2, No. 4, 2016, pp. 57-64. doi: 10.11648/j.jfmhc.20160204.16

Received: June 2, 2016; Accepted: June 20, 2016; Published: November 3, 2016

\begin{abstract}
Intestinal parasitic infection (IPIs) is one of the major and serious medical and public health problems in developing countries including Ethiopia. Effective prevention and control of IPIs require the identification of local risk factors, particularly among school children. The aim of this study was to assess the prevalence of IPIs and associated risk factors among Homesha district school children, Northwest Ethiopia. School based cross sectional study was conducted to from March-June 2015 to achieve this objective. Study subjects were selected by using multi stage sampling technique. A total of 395 school children were enrolled in this study. Socio-demographic data and possible risk factors for the occurrence of IPIs were collected using pre-tested structured questionnaire. Approximately 2 gram of stool specimen was collected and examined for the presence of intestinal parasite using wet mount and formol ether concentration technique. Data were analyzed using SPSS version 20. Chi-square $\left(\chi^{2}\right)$ test and crude odd ratio were calculated to verify and measure the possible association between IPIs and potential risk factors. The overall prevalence of IPs in the present study was 160/395 (35.44\%), for at least one intestinal parasite. Of the nine species of intestinal parasites identified, the most prevalent were $E$. histolytica/dispar $56(14.17 \%)$ followed by G. lamblia $50(12.65 \%)$ and Hook worm $27(10.12 \%)$. Double and triple infections were identified; among these the double and triple infection were $28(20 \%)$ and $6(4.29 \%)$ respectively. In this study the most significantly associated risk factors for the occurrence of IPIs were hand washing habit, eating unwashed/undercooked vegetation, waste disposal habit, shoe wearing habit and practice of figure nail trimming $(\mathrm{P}<0.05)$. Intestinal parasites were prevalent in varying magnitude among the schoolchildren and they are public health problem. Therefore, the woreda health office in collaboration with schools community and other stake holders should work to take measures including education on personal hygiene, environmental sanitation, and treatment should be taken into account to reduce the prevalence of IPIs.
\end{abstract}

Keywords: Associated Risk Factors, Homesha, Intestinal Parasites, Prevalence, Schoolchildren

\section{Introduction}

Intestinal parasitic infection is one of the major and serious medical and public health problems in developing countries. Globally two billion individuals were infected with intestinal parasites; out of these majorities were children in resourcepoor settings [1]. Intestinal parasites are widely distributed in Sub-Saharan Africa including Ethiopia largely due to the lower socio-economic status, poor environmental and personal hygiene, poor nutrition, low literacy rate, overcrowding and climatic conditions that favor the development and survival of these parasites are some of the factors contributing to the high prevalence of intestinal parasitic infection $[2,3,4]$. 
School age children are one of the groups at high-risk for intestinal parasitic infections $[1,5]$. It is estimated that over 270 million pre-school children and over 600 million of school children are living in areas where the parasites are intensively transmitted and are in need of treatment and preventive interventions [6]. According to WHO report, in Ethiopia the two-third proportion of children aged 1-14 years requiring preventive chemotherapy which mainly involve deworming of infected children [2]. Apart from causing morbidity and mortality, infection with intestinal parasites has known to cause iron deficiency leading to poor school performance and absenteeism, growth retardation and other physical and mental health problems in children [7]. This is because the immune systems of children are not yet fully developed and they also habitually play in faecally contaminated soil [8].

A number of studies have been conducted on the distribution and prevalence of intestinal parasites in different parts of Ethiopia [3, 4, 8, 9, 10, 11]. However, the distribution and prevalence of various species of intestinal parasites differ from region to region because of several environmental, social, economical and geographical factors [9]. Hence, the aim of this study was to assess the prevalence of IPIs and associated risk factors among Homesha Woreda school children, Northwest Ethiopia. This study fill the existing gap by providing adequate epidemiological information on the current situation of IPIs prevalence and, local risk factors that give rise to its occurrence in order to identify the mechanisms for transmission. This enable the decision makers, school community and other stake holders to have current information that will be used in improving health status of schoolchildren in Homesha Woreda, Assosa zone. The obtained information is also crucial in evaluating the different intervention strategies that will be implemented in the future.

\section{Materials and Methods}

\subsection{Description of the Study Area}

The study was conducted in Homesha Woreda, Assosa zone in Benishangul-Gumuz regional state, Western Ethiopia. Homesha District is found around $700 \mathrm{kms}$ West of Addis Ababa and around $40 \mathrm{Km}$ far from Assosa town. Assosa zone, one of the three zones in the region, has a total area of 1,519 $\mathrm{Km}^{2}$ and a population of 28,970 (population density of $19.1 / \mathrm{Km}^{2}$ ). The Berta ethnic group are the indigenous population resides mainly in Homesha Woreda and more than $96.3 \%$ of the population of this ethnic group are Muslims. They use farming and mining as source of income to lead their family [12].

\subsection{Study Design and Period}

A school based cross-sectional parasitological study was conducted from March to June 2015 to determine the prevalence of IPIs and associated risk factors.

\subsection{Study Population}

The study population was all schoolchildren found in primary schools of Homesha Woreda. In this Woreda there are around eight elementary schools which have 4214 (2216 male and 1198 female) school children attending from 1 to 8 grade levels.

\subsection{Inclusion and Exclusion Criteria}

Inclusion criteria: Volunteer students and did not took any anti-intestinal parasitic drug within 2 weeks

Exclusion Criteria: Non-volunteers students who took any anti-intestinal parasitic drug within two weeks

\subsection{Sample Size and Sampling Techniques}

The sample size (n) was estimated using the formula for cross-sectional survey [13]:

$\mathrm{n}=\mathrm{Z}^{2} \mathrm{p}(1-\mathrm{p}) / \mathrm{d}^{2}$ Where, $\mathrm{P}=$ Prevalence of intestinal parasites from previous study

$\mathrm{d}=$ Absolute precision and is taken as 0.05 and $\mathrm{Z}=1.96$ at $95 \%$ confidence interval.

$=1.96^{2} \times 0.50 \times 0.50 / .05^{2}$, this gives a sample size of 384 . Since there were no studies conducted concerning the present topic in the area, $\mathrm{p}$ was taken as $50 \%$ to achieve the maximum sample size. To minimize errors arising from the likelihood of non-compliance, five percent of the sample size was added, giving a final sample size of 404 .

Multistage sampling method was used to select the study subjects. All elementary schools in a Woreda were registered first and from the list four schools were selected as a study schools by purposive sampling method. The primary schools selected for this study were Homesha, Tsore Arumela, Tsore Almetema and Shorkele which have 977, 350, 390 and 1050 students attending during data collection. To select the study participant, the students were stratified according to their educational level (grade 1 to grade 8). Classes first selected by simple random sampling technique. Then a quota was allocated for each grade with proportional allocation according to the number of students in each grade. Finally, the participating children were selected using systematic random sampling technique by using class rosters as a sample frame [14].

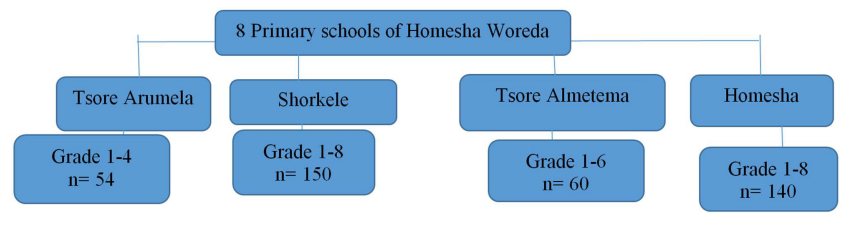

Figure 1. Selection of study participants from Primary schools of Homesha Woreda, Northwest Ethiopia.

\subsection{Methods of Data Collection}

\subsubsection{Interview with Structured Questionnaires}

A structured questionnaire was prepared originally in English according to the research objectives and possible risk factors in the local situation and then was translated in to the local language i.e., Rutanigna. A data collector having 
diploma in clinical nursing was recruited for data collection. To ensure the reliability of the information given during data collection first pilot study was done and the children were interviewed in their mother tongue. The selected students were asked using the revised and pretested structured questionnaires. All the questionnaires were checked for accuracy and completeness.

\subsubsection{Stool Sample Collection and Parasitological Examination}

After proper instruction, the children were given the labeled collection cups and applicator sticks. From each student, about $2 \mathrm{~g}$ of fresh stool was collected. At the time of collection, date of sampling, the name of the participant, age and sex were recorded for each subject on a recording format and checked for its label, quantity and procedure of collection. A portion of each of the stool samples was processed and examined by two senior laboratory technologist microscopically using the following methods [15]. Two different types of laboratory techniques for the examination of stool were employed.

\section{i. Direct Wet Mount Method}

A small portion of stool sample was emulsified with normal saline $(0.85 \% \mathrm{NaCl}$ solution) and placed on a slide. For lugol's iodine staining technique, the stool sample was mixed with a drop of dilute iodine. Cover slip was placed on the emulsion at an angle of $45 \%$ to prevent air bubbles from forming under the cover slip. This method was used to detect the presence of motile intestinal parasites, trophozoite and cysts of protozoan parasites under light microscope at $10 \mathrm{X}$ and $40 \mathrm{X}$ magnification immediately [16]. The remaining part was preserved with $10 \%$ formalin in the ratio of $1 \mathrm{~g}$ of stool to $3 \mathrm{ml}$ of formalin for later examination at Assosa hospital.

\section{ii. Formol-Ether Concentration}

A portion of preserved stool sample was processed by formol-ether concentration method. In brief, using an applicator stick, about $1 \mathrm{~g}$ of preserved stool sample was placed in a clean $15 \mathrm{ml}$ conical centrifuge tube containing 7 $\mathrm{ml}$ formalin. The sample was suspended and mixed thoroughly with applicator stick. The resulting suspension was filtered through a sieve (cotton gauze) into a beaker and the filtrate pour back into the same tube. After adding $3 \mathrm{ml}$ of diethyl ether to the mixture and hand shaken, the content was centrifuged at $2000 \mathrm{rpm}$ for 3 minutes. Iodine stain preparation was made from the sediments. Finally, the entire area under the cover slip were systematically examined using $\times 10$ and $\times 40$ objective lenses and ova or cyst of different parasites can be observed under the microscope [16]. Ten percent of the prepared slides were re-checked by an experienced laboratory technologist.

\subsection{Data Analysis}

The collected data were coded and entered in to SPSS software version 20 to perform the statistical analysis. Chisquare $\left(\chi^{2}\right)$ test was used to verify the possible association between infection and exposure to different factors. Crude odd ratio was also calculated to measure the strength of the association between IPI and potential risk factors. Probability values were considered to be statistically significant when the calculated P-value is equal to or less than 0.05 .

\subsection{Ethical Consideration}

The protocol of the study was reviewed and approved by ethical committee of Assosa University. The study subjects were informed about the objective of the study and, informed assent and consent were obtained from children and guardian of the children, respectively. Confidentiality of all the information was assured.

\section{Results}

\subsection{Socio-demographic Characteristics}

Table 1. Socio-demographic characteristics among schoolchildren of Homesha Woreda, Northwest Ethiopia, March-June 2015.

\begin{tabular}{|c|c|c|c|}
\hline Variables & & Frequency & $\begin{array}{l}\text { Percentage } \\
(\%)\end{array}$ \\
\hline \multirow{3}{*}{ Age in years } & $6-9$ & 237 & 60 \\
\hline & $10-13$ & 133 & 33.7 \\
\hline & $>13$ & 25 & 6.3 \\
\hline \multirow{2}{*}{ Sex } & Male & 207 & 52.4 \\
\hline & Female & 188 & 47.6 \\
\hline \multirow{2}{*}{ Religion } & Christian & 61 & 15.44 \\
\hline & Muslim & 334 & 84.56 \\
\hline \multirow{2}{*}{ Grade } & $1-4$ & 235 & 59.50 \\
\hline & $5-8$ & 160 & 40.50 \\
\hline \multirow{3}{*}{$\begin{array}{l}\text { Mothers } \\
\text { educational } \\
\text { Status }\end{array}$} & Illiterate & 204 & 51.6 \\
\hline & Primary school & 181 & 45.8 \\
\hline & Secondary school and above & 10 & 2.5 \\
\hline \multirow{3}{*}{$\begin{array}{l}\text { Fathers } \\
\text { educational } \\
\text { Status }\end{array}$} & Illiterate & 224 & 56.7 \\
\hline & Primary school & 163 & 41.3 \\
\hline & Secondary school \& above & 8 & 2 \\
\hline
\end{tabular}

A total of 404 schoolchildren were invited to participate and $395(97.77 \%)$ provided proper stool samples and complete information. Among these, 207 (52.40\%) were males and $188(47.59 \%)$ females. The age distribution of the students showed that $237(60 \%)$ students were 9 years old or less, $133(33.7 \%)$ ranged from 10 to 13 years and 25 students $(6.3 \%)$ were above 13 years of age. The mean age of the children was 11.2 years. Of the study participants, 334 $(84.56 \%)$ of them were Muslim and 61 (15.44\%) were Christian by religion. Majority of students were grade 1 to 4 , $235(59.5 \%)$. Majority of the students have illiterate mother $(51.6 \%)$ and illiterate father (56.7\%) (Table 1). 


\subsection{Prevalence of Intestinal Parasitic Infections (IPI)}

Table 2. The prevalence of IPI according to school and sex among Homesha Woreda school children, Northwest Ethiopia, March-June 2015.

\begin{tabular}{lllll}
\hline School Name & Sex & No. of Examined & No. Infected & \% Infected \\
\hline \multirow{3}{*}{ Homosha } & Male & 54 & 18 & 33.33 \\
& Female & 86 & 28 & 32.55 \\
& Sub total & 140 & 46 & 32.85 \\
Tsore Almetema & Male & 37 & 12 & 32.43 \\
& Female & 18 & 12 & 66.66 \\
Tsore Arumela & Sub total & 55 & 24 & 43.63 \\
& Male & 29 & 11 & 37.93 \\
Shomale & Sub total & 21 & 11 & 52.38 \\
& Male & 50 & 22 & 44 \\
& Female & 87 & 25 & 28.73 \\
& Sub total & 63 & 23 & 36.5 \\
& Male & 150 & 48 & 32 \\
& Female & 207 & 66 & 31.88 \\
\end{tabular}

Of the 395 study subjects, 150 (37.97\%), $140(35.44 \%), 55(13.92 \%)$ and $50(12.65 \%)$ students were taken Shorkele, Homesha, Tsore Almetema, and Tsore Arumela respectively. The overall prevalence of IPIs in both sex of the pupils in all schools was $35.44 \%$. Of the 207 male students and 188 female students, 66 (31.88\%) male students and 74 (39.36\%) female students were infected with at least one any intestinal parasites (Table 2).

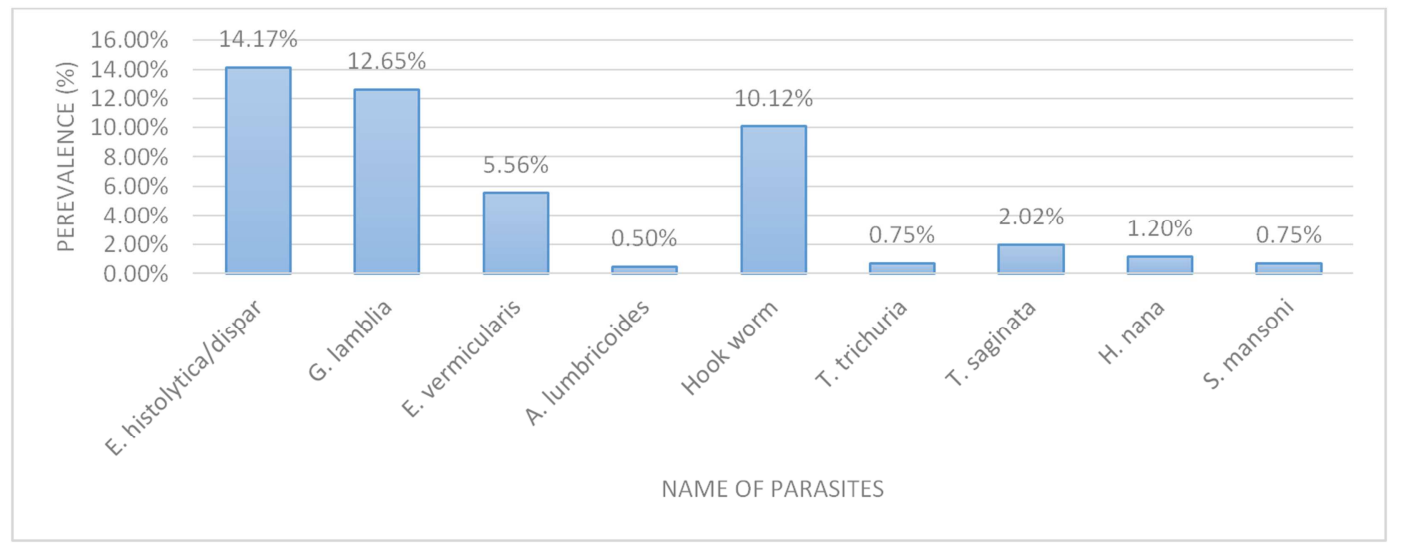

Figure 2. The prevalence of different IPs detected as single or multiple infections among Homesha Woreda school children, Northwest Ethiopia, March-June 2015.

The most prevalent intestinal parasites identified were E. histolytica/dispar 56 (14.17\%) followed by G. lamblia 50 (12.65\%) and Hook worm 40 (10.12\%). E. vermicularis, T. saginata, H. nana, S. mansoni and T. trichuria and A. lumbricoides were also detected as single or multiple infections in $11(5.56 \%), 8(2.02 \%), 5(1.2 \%), 3(0.75 \%)$ and $2(0.5 \%)$ individuals, respectively (Fig. 2).

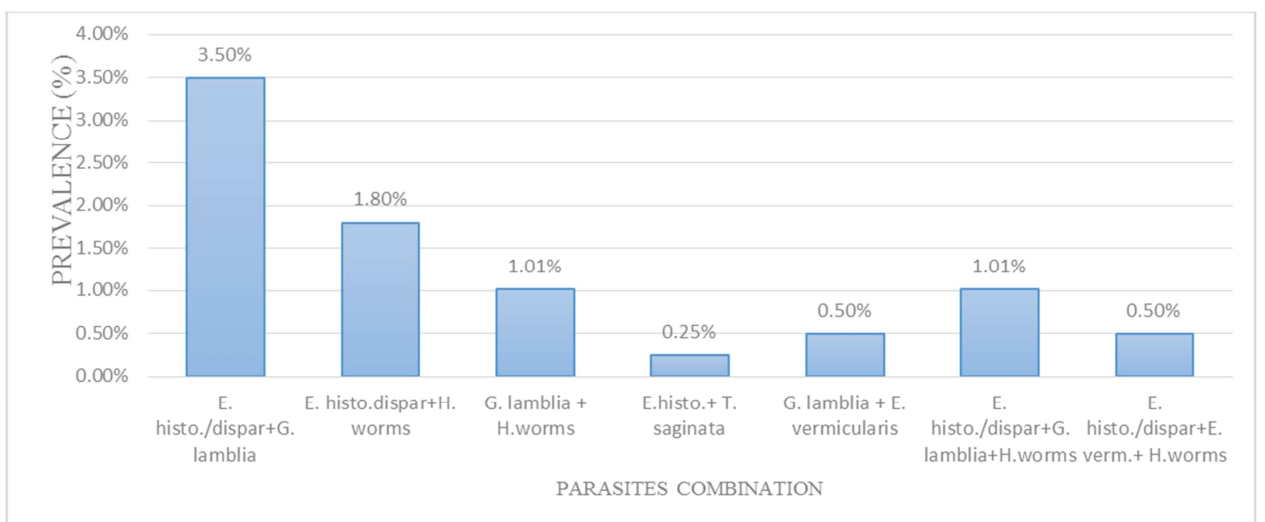

Figure 3. Parasites combination for mixed intestinal parasite infection among Hemosha Woreda schoolchildren, Northwest Ethiopia, March-June 2015. 
Different parasites combine to cause mixed infection that can be either double or triple infection. The most common combination for double infection were E. histolytica/dispar + G. lamblia 14 (3.5\%), E. histolytica/dispar $+\mathrm{H}$. worms 7 (1.8\%) and $G$. lamblia $+\mathrm{H}$. worms $4(1 \%)$. The most common combination for triple infection were E. histolytica + G. lamblia $+\mathrm{H}$. worms 4 (1.01) and E. histolytica + E. vermicularis + H. worms (Fig.-3).

\subsection{Association of IPIs with Potentially Associated Risk Factors}

Table 3. Association of IPIs with socio-demographic factors among Homesha Woreda schoolchildren, Northwest Ethiopia, March-June 2015.

\begin{tabular}{|c|c|c|c|c|c|}
\hline \multirow{2}{*}{ Risk factors } & & \multicolumn{2}{|l|}{ Intestinal parasites } & \multirow{2}{*}{$\mathbf{X}^{2}$} & \multirow{2}{*}{ P-value } \\
\hline & & No. Examined (\%) & No. infected (\%) & & \\
\hline \multirow{3}{*}{ Age } & $6-9$ & $237(60)$ & $88(37.13)$ & \multirow{3}{*}{170.69} & \multirow{3}{*}{0.01} \\
\hline & $10-13$ & $133(33.7)$ & $50(37.59)$ & & \\
\hline & $>13$ & $25(6.3)$ & $2(8)$ & & \\
\hline \multirow{2}{*}{ Sex } & Male & $207(52.4)$ & $67(32.36)$ & \multirow{2}{*}{.914} & \multirow{2}{*}{0.08} \\
\hline & Female & $188(47.6)$ & $73(38.82)$ & & \\
\hline \multirow{2}{*}{ Grade } & $1-4$ & $235(59.50)$ & $92(39.14)$ & \multirow{2}{*}{162} & \multirow{2}{*}{0.00} \\
\hline & $5-8$ & $160(40.50)$ & $48(30)$ & & \\
\hline \multirow{3}{*}{ Mothers educational Status } & Illiterate & $204(51.6)$ & $131(64.21)$ & \multirow{3}{*}{170.64} & \multirow{3}{*}{0.21} \\
\hline & Primary school & $181(45.8)$ & $6(3.31)$ & & \\
\hline & Secondary school \& above & $10(2.5)$ & $3(3)$ & & \\
\hline \multirow{3}{*}{ Fathers educational Status } & Illiterate & $224(56.7)$ & $132(58.92)$ & \multirow{3}{*}{188.35} & \multirow{3}{*}{0.86} \\
\hline & Primary school & $163(41.3)$ & $7(4.2)$ & & \\
\hline & Secondary school \& above & $8(2)$ & $1(12.5)$ & & \\
\hline
\end{tabular}

Sex and mother and father educational status were not statistically associated with IPIs (P>0.05). Age and grade were statistically associated with IPIs $(\mathrm{P}<0.05)$ (Table 3$)$.

Table 4. Association of IPIs with behavioral and sanitary habits among Homesha Woreda school children, Northwest Ethiopia, March-June 2015.

\begin{tabular}{|c|c|c|c|c|c|}
\hline \multirow{2}{*}{ Risk factors } & & \multicolumn{2}{|l|}{ Intestinal parasites } & \multirow{2}{*}{$\mathbf{X}^{2}$} & \multirow{2}{*}{ P-value } \\
\hline & & No. Examined (\%) & No. infected (\%) & & \\
\hline \multirow{2}{*}{ Hand washing habit before eating and after defecation } & Yes & $108(27.34)$ & $18(16.66)$ & \multirow{2}{*}{81.11} & \multirow{2}{*}{0.023} \\
\hline & No & $287(72.66)$ & $122(42.5)$ & & \\
\hline \multirow{2}{*}{ Habit of eating raw meat } & Yes & $55(13.9)$ & $51(92.72)$ & \multirow{2}{*}{205.6} & \multirow{2}{*}{0.543} \\
\hline & No & $340(86.1)$ & $89(26.1)$ & & \\
\hline \multirow{2}{*}{ Habit of eating unwashed/undercooked vegetables } & Yes & $133(33.7)$ & $125(93.98)$ & \multirow{2}{*}{42.12} & \multirow{2}{*}{0.011} \\
\hline & No & $262(66.3)$ & $15(5.7)$ & & \\
\hline \multirow{2}{*}{ Shoe wearing habit } & Yes & $226(57.21)$ & $19(8.4)$ & \multirow{2}{*}{8.225} & \multirow{2}{*}{0.001} \\
\hline & No & $169(42.78)$ & $121(71.59)$ & & \\
\hline \multirow{2}{*}{ Swimming habit } & Yes & $82(20.75)$ & $63(76.82)$ & \multirow{2}{*}{135.0} & \multirow{2}{*}{0.635} \\
\hline & No & $313(79.25)$ & $77(24.6)$ & & \\
\hline \multirow{2}{*}{ Habit of fingernail trimming } & Yes & $170(43.03)$ & $19(11.17)$ & \multirow{2}{*}{8.225} & \multirow{2}{*}{0.007} \\
\hline & No & $225(56.97)$ & $121(53.77)$ & & \\
\hline \multirow{3}{*}{ Water source } & Pipe & $370(93.7)$ & $121(32.7)$ & \multirow{3}{*}{993.48} & \multirow{3}{*}{0.984} \\
\hline & Spring & $7(1.8)$ & $5(71.4)$ & & \\
\hline & River & $18(4.5)$ & $14(77.77)$ & & \\
\hline \multirow{2}{*}{ Toilet } & Present & $373(94.43)$ & $124(33.24)$ & \multirow{2}{*}{311.9} & \multirow{2}{*}{0.712} \\
\hline & Absent & $22(5.56)$ & $16(72.72)$ & & \\
\hline \multirow{3}{*}{ Waste disposal } & Burry underground & $307(77.72)$ & $73(18.48)$ & \multirow{3}{*}{613.7} & \multirow{3}{*}{0.005} \\
\hline & Open field & $72(18.22)$ & $57(79.16)$ & & \\
\hline & Incirinate & $16(4.05)$ & $10(17.54)$ & & \\
\hline
\end{tabular}

From the total respondents that had no habit of hand washing before meal and after defecation were 287 (72.66\%). From the total respondents, respondents that had no habit of eating raw meat were $340(86.1 \%)$. The association between eating raw meat and habit of swimming, and IPI was not significant statistically $(\mathrm{P}<0.05)$. The majority of the students $370(93.7 \%)$ used pipe water as a source of drinking water. The relation between water source and IPIs was not statistically associated ( $\mathrm{P}>0.05)$. (Table-4). 


\subsection{Crude Associations of Independent Variables with IPI}

Table 5. Crude odd ratios of IPIs and significantly associated risk factors among Homesha Woreda school children, Northwest Ethiopia, March-June 2015.

\begin{tabular}{|c|c|c|c|c|c|}
\hline \multirow{2}{*}{ Risk factors } & & \multicolumn{2}{|l|}{ Intestinal parasites } & \multirow{2}{*}{$\begin{array}{l}\text { Crude OR (95\% } \\
\text { CI) }\end{array}$} & \multirow{2}{*}{ P-value } \\
\hline & & No. Examined (\%) & No. infected (\%) & & \\
\hline \multirow{3}{*}{ Age in years } & $6-9$ & $237(60)$ & $88(37.13)$ & \multirow{3}{*}{$3.51(2.31-5.81)$} & \multirow{3}{*}{0.01} \\
\hline & $10-13$ & $133(33.7)$ & $50(37.59)$ & & \\
\hline & $>13$ & $25(6.3)$ & $2(8)$ & & \\
\hline \multirow{2}{*}{ Grade } & $1-4$ & $235(59.50)$ & $92(39.14)$ & \multirow{2}{*}{$4.20(3.34-8.50)$} & \multirow{2}{*}{0.00} \\
\hline & $5-8$ & $160(40.50)$ & $48(30)$ & & \\
\hline \multirow{2}{*}{$\begin{array}{l}\text { Hand washing habit before eating and after } \\
\text { defecation }\end{array}$} & Yes & $108(27.34)$ & $18(16.66)$ & \multirow{2}{*}{$5.45(4.55-11.90)$} & \multirow{2}{*}{0.023} \\
\hline & No & $287(72.66)$ & $122(42.5)$ & & \\
\hline \multirow{2}{*}{$\begin{array}{l}\text { Habit of eating unwashed/undercooked } \\
\text { vegetables }\end{array}$} & Yes & $133(33.7)$ & $125(93.98)$ & \multirow{2}{*}{$0.312(0.015-0.063)$} & \multirow{2}{*}{0.011} \\
\hline & No & $262(66.3)$ & $15(5.7)$ & & \\
\hline \multirow{2}{*}{ Shoe wearing habit } & Yes & $226(57.21)$ & $19(8.4)$ & \multirow{2}{*}{$2.3(1.2-4.67)$} & \multirow{2}{*}{0.001} \\
\hline & No & $169(42.78)$ & $121(71.59)$ & & \\
\hline \multirow{2}{*}{ Habit of fingernail trimming } & Yes & $170(43.03)$ & $19(11.17)$ & \multirow{2}{*}{$0.212(0.162-0.276)$} & \multirow{2}{*}{0.007} \\
\hline & No & $225(56.97)$ & $121(53.77)$ & & \\
\hline \multirow{3}{*}{ Waste disposal habit } & Burry underground & $307(77.72)$ & $73(18.48)$ & \multirow{3}{*}{$1.88(1.12-4.42)$} & \multirow{3}{*}{0.005} \\
\hline & Open field & $72(18.22)$ & $57(79.16)$ & & \\
\hline & Incirinate & $16(4.05)$ & $10(17.54)$ & & \\
\hline
\end{tabular}

The crude odd ratio for age, grade, hand washing before eating and after defecation, practice of eating unwashed/undercooked vegetables, wearing shoes, practice of fingernail trim, and waste disposal were 3.51, 4.20, 5.45, $0.312,2.3,0.212$ and 1.88 respectively. From the potentially associated risk factors, age, grade, hand washing before eating and after defecation, practice of eating unwashed/undercooked vegetables, wearing shoes, practice of fingernail trim, and waste disposal were significantly associated $(\mathrm{p}<0.05)$ (Table 5).

\section{Discussion}

The present study attempted to assess the prevalence of different IPIs among schoolchildren of Homesha Woreda, Northwest Ethiopia. The observed prevalence of intestinal parasites of $140(35.44 \%)$ is almost similar with study conducted at the University of Gondar Community School, Northwest Ethiopia [3]. They reported about $34.2 \%$ of the schoolchildren were infected with one or more intestinal parasites. Ref. 17 also reported almost similar but slightly higher prevalence rate (37.8\%) from Chelaleki Health Center and Red Cross Clinic, Eastern Wellega.

The observed prevalence of intestinal parasites in this study was lower compared with reports of other similar studies, $60.7 \%$ in Wukro town, Tigray [18], 77.9\% in Dagi primary school, Amhara Region [11], 84.3\% in Debre Elias Primary Schools, East Gojjam Zone [8], 79.8\% in Delgi school children, North Gondar [19]. On the other hand, the prevalence observed in this study was higher than a study conducted in Babile [20].

The contradictory report on the prevalence of IPI could be due to variation in awareness regarding transmission and prevention of IPs between study participants in this study and previous studies. In addition, the occurrence of diversified IPs might be due to poor environmental and personal hygiene, habit of walking on bare foot, lack of education which increases overall magnitude of IPs. On the other hand, as the collection period was short, potential seasonal fluctuations might have affected the actual prevalence. Modified acid-fast staining technique was not used to detect Cryptosporidium species. Moreover, we conducted single stool examination for detection of intestinal parasites, which could have underestimated the prevalence.

In the present study E. histolytica/dispar 56 (14.17\%) was the most predominant parasite followed by G. lamblia 50 $(12.65 \%)$, Hook worm $27(10.12 \%)$. This is almost similar to a finding from a study conducted in Chelaleki Health Center and Red Cross Clinic, Eastern Wellega, E. histolytica/dispar $60(16.3 \%)$ was the most predominant parasite followed by G. lamblia 33 (9\%) and Hookworm 14 (3.8\%) [17]. In contrary to this study, the research conducted in Debre Elias Primary Schools, East Gojjam Zone by ref. 8, reported E. histolitica/dispar with prevalence of $6.7 \%$. This could be due to difference in environmental and living conditions of the study participants. The high occurrence of the parasitic protozoan infections in the present study may be due to poor environmental sanitation (including poor waste disposal habit), which favor the persistence of cyst of the parasites in the soil.

In the present study, $H$. nana, constituted about 5 (1.2\%). This finding was lower than the study conducted in Debre Elias, East Gojjam (1.8\%) [8] and Babile (10.1\%) [20]. However, the finding was higher than studies conducted in East Wollega (0.3\%) [17]. The possible explanation for this difference might be due to the time gap, geographical and environmental difference of the localities.

The present study also assessed the possible association of intestinal parasite infection with potential risk factors among school children. Several recent studies have identified a range of environmental, behavioral and social risk factors associated with intestinal parasite infections $[9,3,4,8,10$, 
$11,17,18,19]$. However, some of them were significantly associated in this study making it comparable to earlier studies in Ethiopia and other less developed countries and explained as follow:

Intestinal parasitic infections were significantly associated with poor hand washing practice and habit of eating unwashed/undercooked vegetables $(\mathrm{p}<0.05)$. The likelihood of acquiring infections among students who do not practice hand washing before eating and after defecation was 5.45 times higher than among those who had good hand washing practice. Moreover, the likelihood of acquiring infections among students who had the habit of eating unwashed/undercooked vegetables was 0.312 times higher. This is probably due to low knowledge of schoolchildren about the feco-oral transmission of intestinal parasite through their unwashed hands. In addition to this contamination of vegetables with fecal materials in the farm and contamination of the vegetation with wastes in home produce conducive environment for transmission intestinal protozoa.

In this study, the level of education of the study participants and their age were significantly associated with intestinal parasitic infections $(\mathrm{P}<0.05)$. This is similar to a report in Zarima town [5] and Dagi primary school [11]. The likelihood of acquiring infections among students who were under $4^{\text {th }}$ grade and age of 6-9 were 4 and 3.5 times higher as compared to the other students. The possible explanation might be the level of awareness about washing hands, playing in the open fields and other personal hygiene measures in children whose grade become (1-4) was lower than those whose grade become 5-8. This causes passing viable ova to one another when they use dirty hands to share foods. The other reason might be those students whose grade from 1 to 4 was less immunized compared to grade 5 to 8 . Thus, as age increases the prevalence of parasitic infection decreases possibly due to improved personal hygiene, reduced contact with soil and developing immunity against parasites.

Though IPIs were not significantly associated with sex, the prevalence is higher in female students (39.36\%) as compared to male students $(31.88 \%)$. This is so because female children are more actively involved in carrying out activities in and out of their immediate environment thereby exposing them to infection.

\section{Conclusion and Recommendation}

The findings of the present study showed IPIs were prevalent health problem among school children of Homesha Woreda. Public education program on personal hygiene, improved sanitation including proper waste disposal, avoiding eating unwashed/undercooked vegetation, proper nail trimming and wearing shoes should be provided to prevent and reduce the prevalence of IPIs. In addition to this, regular monitoring of these practices by school director and teachers is also suggested. Further detailed investigation by using the Kato-katz method should be conducted to determine the intensity of schistosomiasis in the area.

\section{References}

[1] WHO, "The prevention and control of Schistosomiasis and soil transmitted Helminthiasis. Geneva" WHO; 2002

[2] WHO, "Eliminating soil-transmitted helminthiases as a public health problem in children. Progress report 2001-2010 and strategic plan 2011-2020," 2012.

[3] A. Gelaw, B. Anagaw, B. Nigussie, B. Silesh, A. Yirga, M. Alem, M. Endris, and B. Gelaw, "Prevalence of intestinal parasitic infections and risk factors among schoolchildren at the University of Gondar Community School, Northwest Ethiopia: a cross-sectional study," BMC Public Health, vol. 13, 304-311, 2013.

[4] T. Wegayehu, T. Tsalla, B. Seifu, and T. Teklu, "Prevalence of intestinal parasitic infections among highland and lowland dwellers in Gamo area, South Ethiopia," BMC Public Health., vol. 13, 151-158, 2013.

[5] A. Alemu, A. Atnafu, Z. Addis, Y. Shiferaw, T. Teklu, B. Mathewos, W. Birhan, S. Gebretsadik, and B. Gelaw, "Soil transmitted helminths and schistosoma mansoni infections among school children in zarima town, northwest Ethiopia," BMC Infectious Diseases, vol. 11, 189-196, 2011.

[6] WHO, "Soil-transmitted helminthiasis. Number of children treated 2007-2008: update on the 2010 global target," Wkly Epidemiol Rec, vol. 85, 141-14, 2010.

[7] M. O. Harhay, J. Horton, and P. L. Olliaro, "Epidemiology and control of human gastrointestinal parasites in children," Expert Rev Anti Infect Ther, vol. 8, 219-234, 2010.

[8] T. Workneh, A. Esmael, and M. Ayichiluhm, "Prevalence of Intestinal Parasitic Infections and Associated Factors among Debre Elias Primary Schools Children, East Gojjam Zone, Amhara Region, North West Ethiopia," J Bacteriol Parasitol, vol. $5,1-6,2014$.

[9] M. Legesse, and B. Erko, "Prevalence of intestinal parasites among schoolchildren in a rural area close to the southeast of Lake Langano, Ethiopia," Ethiop. J. Health Dev., vol. 18, 116$120,2004$.

[10] E. Tigabu, B. Petros, and T. Endeshaw, "Prevalence of Giardiasis and Cryptosporidiosis among children in relation to water sources in Selected Village of Pawi Special District in Benishangul-Gumuz Region, Northwestern Ethiopia,” Ethiop. J. Health Dev., vol. 24, 205-213, 2010.

[11] M. Alamir, W. Awoke and A. Feleke, "Intestinal parasites infection and associated factors among schoolchildren in Dagi primary school, Amhara National Regional State, Ethiopia," Health, 5, 1697-170, 2013.

[12] T. Flatie, T. Gedif, K. Asres and T. Gebre-Mariam, "Ethnomedical survey of Berta ethnic group Assosa Zone, Benishangul-Gumuz regional state, mid-west Ethiopia," Journal of Ethnobiology and Ethnomedicine, vol. 5, 14-25, 2009.

[13] W. Daniel, Biostatistics: A foundation for analysis in the health sciences. 6th ed. New York: Willey. 1995

[14] M. Alemu, A. Hailu, and G. Buggsa, "Prevalence of intestinal Schistosomiasis and soil transmitted heliminthiasis among primary school children in umolante district, South Ethiopia," Clinical Medicine Research, 3960, 174-180, 2014. 
[15] K. D. Parameshwarappa, C. Chandrakanth, and B. Sunil, "The prevalence of intestinal parasitic infestations and the evaluation of different concentration techniques of the stool examination," J. Clin. Diagnostic Res., 4662, 2392-2396, 2012.

[16] T. Kiran, N. Shashwati, B. Vishal, and D. A. Kumar "Intestinal Parasitic infections and Demographic status of schoolchildren in Bhopal region of Central India," Journal of Pharmacy and Biological Sciences, 9, 83-87, 2014.

[17] A. A. Gebru, B. A. Bizune, Y. Y. Ayene, Z. M. Semene, A. W. Hailu, T. Nigussie, and M. K. Assefa, " Prevalence of Intestinal Parasites and Associated Risk Factors at Red Cross Clinic and Chelaleki Health Center, East Wollega Zone, Ethiopia," Science Journal of Public Health, 3, 445-452, 2015.
[18] E. Kidane, S. Menkir, A. Kebede and M. Desta, "Prevalence of intestinal parasitic infections and their associations with anthropometric measurements of school children in selected primary schools, Wukro Town, Eastern Tigray, Ethiopia," Int. J. Curr. Microbiol. App., 3, 11-29, 2014.

[19] A. Asrat, D. Tewodros, and W. Alemayehu, "Prevalence and risk factors of IPs among Delgi school children, Northern Gonder, Ethiopia," Journal of Parasitology and Vectoriology, 3, 75-81, 2011.

[20] G. I. Tadesse, "Prevalence of intestinal helminthic infection associated risk factors among school children in Babile town eastern Ethiopia,” Ethiop. J Health Dev., 19, 140-147, 2005. 